\title{
Depresión posparto: ¿se encuentra asociada a la violencia basada en género?
}

\author{
Post partum depression: is it associated to gender based violence?
}

\author{
Juan Escobar ${ }^{1}$, Percy Pacora ${ }^{2}$, Nilton Custodio ${ }^{3}$, Walter Villar ${ }^{1}$ \\ ${ }^{1}$ Médico Cirujano, Universidad Nacional Mayor de San Marcos. Lima, Perú. \\ ${ }^{2}$ Médico Asistente del Hospital Docente Madre Niño San Bartolomé. Lima, Perú. \\ ${ }^{3}$ Médico Neurólogo, Universidad Nacional Mayor de San Marcos. Lima, Perú.
}

\section{Resumen}

Introducción: La depresión posparto y la violencia basada en género son patologías muy frecuentes en la mujer; la importancia de un diagnóstico y tratamiento oportuno radica en evitar las consecuencias a futuro en la mujer, el recién nacido y la familia en general. Objetivos: Determinar la asociación entre depresión posparto y la violencia basada en género. Diseño: Exploratorio, de corte transversal. Lugar: Hospital Nacional Docente Madre Niño San Bartolomé, Lima, Perú. Participantes: Mujeres puérperas. Intervenciones: Se aplicó una ficha de datos sociodemográficos y del parto, la escala de Edimburgo, el inventario revisado de factores predictores de depresión posparto y el instrumento de detección de violencia basada en género a 100 mujeres, de 1 a 12 meses de tiempo puerperal. Principales medidas de resultados: Asociación entre depresión posparto y violencia basada en género. Resultados: La tasa de depresión posparto fue $17 \%$, encontrando diferencias estadísticas al compararla con autoestima, depresión previa al embarazo, ansiedad durante la gestación, satisfacción marital y eventos estresantes durante la gestación. El $51 \%$ fue víctima de violencia basada en género, siendo la más frecuente la forma psicológica. Se halló diferencias estadísticas entre depresión posparto y violencia basada en género $(p=0,001)$, así como, diferencias estadísticas entre depresión posparto y violencia durante la gestación, encontrando un $\mathrm{OR}$ de 5,5 (IC: < 5,5; 16,7>). Conclusiones: Las mujeres víctimas de violencia tienen riesgo elevado de sufrir depresión posparto.

Palabras clave: Depresión posparto; violencia basada en género; autoestima; ansiedad.

\begin{abstract}
Introduction: Post partum depression and gender based violence are very common disorders in women; the importance of early diagnosis and prompt treatment is to avoid future consequences in women, newborns and the family. Objectives: To determine the association between post partum depression and gender based violence. Design: Exploratory cross-sectional. Place: Hospital Nacional Docente Madre Niño San Bartolome, Lima, Peru. Participants: Post partum women. Interventions: We applied a sociodemographic data and delivery card, the Edinburgh scale, revised inventory predictors of post partum depression and an instrument for detection of gender based violence to 100 women 1 to 12 months post partum. Data analysis was conducted using odds ratio tests, Chi-square and Fisher exact test. Main outcome measures: Association between post partum depression and gender based violence. Results: The rate of post partum depression was $17 \%$ with statistical difference between the cases of post partum depression and self-esteem, depression before pregnancy, anxiety during pregnancy, marital satisfaction and stressful events during pregnancy. Moreover, $51 \%$ of women were victims of gender based violence, mainly psychological and the husband being the principal aggressor. The cases of gender based violence and post partum depression were found significantly different $(p=0,001)$. Also, statistical differences were found between depression post partum y gender based violence during pregnancy, $\mathrm{OR} 5,5$ ( $\mathrm{Cl}:<5,5 ; 16,7>)$. Conclusions: There is increased risk of depression post partum in women victims of violence.
\end{abstract}

Key words: Depression, postpartum; violence against women; self concept; anxiety.

\section{INTRODUCCIÓN}

La depresión es un trastorno del humor que compromete todos los sistemas del organismo, afecta a todas las edades, razas, géneros y momentos de la persona ${ }^{(1-3)}$. Al comparar las prevalencias de depresión entre hombres y mujeres, se evidencia en la niñez cifras equivalentes y, en la adultez, es más frecuente en mujeres ${ }^{(4-6)}$. Esta diferencia se observa fundamentalmente en la etapa reproductiva de la mujer y se asocia con el síndrome premenstrual, embarazo, puerperio y menopausia ${ }^{(3,4,7)}$.

En el periodo puerperal, definido de acuerdo a su duración como inmediato (periodo comprendido entre el parto y las 24 horas del posparto), mediato (comprendido entre el parto y los 42 primeros días) y tardío (entre el parto y el año posparto), se observa importantes variaciones fisiológicas, debido al declive posparto de las hormonas reproductivas (8-10), lo cual podría condicionar a la mujer a sufrir depresión, definida según el Manual Estadístico de Enfermedades Mentales versión IV (DSM-IV) como la presentación de depresión a partir de las 4 semanas posteriores al parto ${ }^{(11-13)}$.

Existen factores de riesgo biológicos. como los trastornos del humor y ansiedad, depresión posparto anterior, síndrome premenstrual disfórico e historia familiar de enfermedades psiquiátricas; y psicosociales, como historia de abusos cometidos durante la niñez, adolescencia, embarazo no planificado, sentimientos negativos de la gestación, madre soltera, gran número de hijos, inadecuado soporte económico, violencia marital, nivel edu- cativo bajo y abuso de sustancias, como alcohol y tabaco ${ }^{(14-16)}$. En investigaciones comparativas, se ha observado que los factores psicosociales son los de mayor importancia en la presentación de depresión posparto, por lo que son llamados factores de riesgo mayores ${ }^{(17-19)}$. Esta conjunción de factores y cambios hormonales hacen que la depresión sea una de las enfermedades más frecuentes del puerperio, encontrándose en diferentes estudios una prevalencia que oscila entre 10 y $20 \%(20-22)$.

El diagnóstico de depresión posparto es clínico y dentro de los síntomas encontramos tendencia al llanto, ánimo sombrío, pérdida de satisfacción por las cosas, rechazo social, insomnio, pérdida de apetito o incremento de apetito, disminución de la concentración, síntomas 
de desesperanza, apatía e infelicidad y síntomas de culpa al no poder cuidar al bebé; llegando a presentarse síntomas somáticos, tales como dolor tipo quemazón, cefalea y dolores de espalda ${ }^{(23-25)}$.

Asimismo, algunos estudios describen que el maltrato físico, psíquico y sexual, la privación de libertad o la carencia de soporte socioeconómico de la mujer se relacionan con la presentación de cuadros depresivos ${ }^{(26,27)}$. Esta gama de agresiones son conocidas como violencia basada en género, considerada como un serio problema de salud pública, por su frecuencia y el impacto que tiene tanto en la mujer como en la familia ${ }^{(25,27)}$.

Se define, según la ONU, como todo acto de violencia basado en género que tiene como resultado posible o real un daño físico, sexual o psicológico, incluidas amenazas, coerción o privación arbitraria de la libertad ${ }^{(26)}$. La Organización Panamericana de la Salud estima que 20 a $60 \%$ de las mujeres que viven en las Américas sufren de situaciones de violencia ${ }^{(25,26)}$. En América del Sur; se estima que 25\% de las mujeres sufre regularmente violencia intrafamiliar y que $50 \%$ pasará por alguna situación de violencia a lo largo de su vida ${ }^{(26,27)}$. En Lima, se detectó que $61 \%$ de las mujeres en general y $54 \%$ de las embarazadas en algún momento de su vida había sido victima de algún tipo de violencia, siendo la violencia psicológica la más frecuente ${ }^{(25,28)}$. Las consecuencias de la violencia basada en género pueden ser fatales, como incremento del número de homicidios, suicidios y de la tasa de mortalidad materna; y no fatales, como trastornos somáticos crónicos, de salud mental y de salud reproductiva, las cuales comprometen tanto a la madre como al bebé, encontrando que las mujeres víctimas de violencia presentan mayor probabilidad de presentar embarazos complicados, muerte fetal, hijos con peso bajo al nacer, parto prematuro, preeclampsia, restricción del crecimiento intrauterino y depresión respiratoria del neonato ${ }^{(29-31)}$.

Teniendo el conocimiento y la implicancia de la depresión posparto y violencia basada en género en la madre, el bebé, la familia y la sociedad, es que se planteó la realización del presente estudio, buscando describir la asociación entre dichas variables en una población de Lima.

\section{MÉTODOS}

Se realizó un estudio observacional de corte transversal en el Hospital Nacional Docente Madre Niño San Bartolomé, perteneciente al Ministerio de Salud del Perú, durante los meses de febrero y marzo de 2008.

El número de mujeres evaluadas fue 100 y el tipo de muestreo realizado fue no probabilístico. Se consideró como criterios de inclusión a las mujeres que se encontraban entre el mes y el año de puerperio, que accedieron a participar en el estudio, firmaron el consentimiento informado; y, como criterios de exclusión, a las mujeres con diagnóstico previo de depresión, síndrome de Sheehan, hipotiroidismo, sordera, ceguera, enfermedad psiquiátrica. Se seleccionó a las madres que acudieron al control de su niño en los consultorios de pediatría de crecimiento y desarrollo y de niño de riesgo alto. El grupo de madres perteneció al grupo temporal de puerperio de 1 a 12 meses, debido a que el DSM-IV describe que la depresión posparto se presenta en dicho período. A las madres que accedieron participar se les solicitó, previa firma de consentimiento informado, contestar un cuestionario de datos generales y del parto, la escala de depresión posparto de Edimburgo, el inventario revisado de Beck y el instrumento de detección de violencia de género 8 . El diagnóstico de depresión posparto se realizó mediante la aplicación de la escala de Edimburgo, la cual consiste en un conjunto de 10 preguntas de síntomas depresivos enfocados al periodo posnatal, validada en Perú por Vega y col. ${ }^{(32-34)}$. Se consideró que una puérpera que sufría de depresión presentaba una puntuación mayor o igual a 13 puntos. Factores como autoestima, depresión y ansiedad, antes y durante la gestación, tristeza posparto y aspectos relacionados al cuidado del recién nacido fueron evaluados mediante el inventario revisado de Beck. Con respecto al diagnóstico de violencia, se empleó el instrumento de detección de violencia basada en género diseñado por la Fede- ración Latinoamericana de Sociedades de Obstetricia y Ginecología (FLASOG); la cual ha sido utilizada en nuestro medio por Tavara y col. ${ }^{(31)}$ y detecta violencia física, psicológica, sexual y abuso durante la infancia.

El procesamiento de los datos se realizó mediante estadística descriptiva en el caso de variables cualitativas, estadística inferencial para el caso de variables cuantitativas, la prueba $\mathrm{X}^{2}$ para las variables cualitativas y se utilizó la medida odds ratio $(\mathrm{OR})$ para determinar la asociación existente entre las variables principales. Se consideró valores de $p<0,05$ como datos significativos.

\section{RESULTADOS}

Se registró los datos generales de la gestación, el parto y puerperio de la totalidad de participantes, según se muestra en la tabla.

En relación al estado afectivo de la madre, $4 \%$ presentó autoestima baja, $27 \%$ manifestó haberse sentido deprimida antes del embarazo, $72 \%$ dijo haberse sentido deprimida, $47 \%$ ansiosa durante la gestación y $51 \%$ haber presentado tristeza posparto.

Con respecto al soporte social recibido por la pareja, se encontró que era adecuado en $82 \%$ de los casos y $42 \%$ manifestó encontrarse insatisfecha con su actual estado civil o con su pareja. Del total de participantes del estudio, se encontró que $18,5 \%$ y $34 \%$ habían presentado problemas con el cuidado y el comportamiento del bebé, respectivamente.

Posteriormente a la recolección de datos, se detectó que 17\% de las mujeres presentó depresión posparto; los puntajes obtenidos oscilaron entre 0 y 25 puntos, con una media y moda de $7,5 \pm 5$ y 10 puntos, respectivamente.

Las relaciones entre los casos depresión posparto y estado civil, nivel socioeconómico, nivel educativo, planificación del embarazo y tipo de parto no fueron estadísticamente significativas. Las variables relacionadas al estado anímico durante la gestación -como autoestima $(p=0,001)$, ansiedad durante la gestación $(p=0,001)$, historia de depresión previa al emba- 
Tabla. Características generales de puérperas estudiadas.

\begin{tabular}{lc}
\hline Edad & $X=27,8$ años \\
Tiempo de puerperio & $X=4,5$ meses \\
Estado civil & Número de puérperas \\
Soltera & 12 \\
Casada & 32 \\
Conviviente & 56 \\
Viuda & 0 \\
Planificación del embarazo & Porcentaje \\
Sí & $34 \%$ \\
No & $66 \%$ \\
Tipo de parto & Porcentaje \\
Vaginal & $74 \%$ \\
Cesárea & $26 \%$ \\
\hline
\end{tabular}

razo $(p=0,012)$, la satisfacción marital $(p=0,036)$, los eventos estresantes durante la gestación $(p=0,041)$ y los problemas con el cuidado del bebé $(p=0,041)$ fueron estadísticamente significativos en relación a los cuadros de depresión posparto, al aplicar la prueba chi-cuadrado. Asimismo, la medida de asociación OR entre depresión posparto y ansiedad durante la gestación $(\mathrm{OR}=7,1 ; \mathrm{IC}:<1,9 ; 26,6>)$, historia de depresión previa al embarazo $(\mathrm{OR}=4,06$; IC: $<0,11 ; 0,97>)$ y problemas por los cuidados del bebé $(\mathrm{OR}=4,1$; IC: $\langle 1,3 ; 1,2\rangle)$ mostraron que existe mayor probabilidad de sufrir depresión en aquellas puérperas que presentan dichos antecedentes. No se encontró diferencias estadísticas ni asociación con depresión durante la gestación, soporte social de la pareja, problemas con el comportamiento del bebé ni con tristeza materna.

Con respecto a las cifras de casos de violencia basada en género se encontró que $51 \%$ de las participantes fue víctima de diferentes tipos de violencia, en algún momento de sus vidas; de ellas, $27 \%$ manifestó haberla sufrido durante el embarazo, $45 \%$ fue víctima de violencia psicológica, $15 \%$ de violencia física, $13 \%$ de violencia sexual durante la niñez y $10 \%$ en edades posteriores. Asimismo, se detectó que a pesar que en la mayoría de casos el principal agresor fue la pareja, paradójicamente 91\% de las mujeres afirmó sentirse segura cuando está en casa con él.

En relación a las variables principales del estudio, se halló diferencias significativas entre violencia y depresión posparto $(p=0,001)$. Se calculó la asociación para dichas variables, con un valor de $\mathrm{OR}=$ 9,8 (IC: $<2,1 ; 45>$ ), lo cual indica que las mujeres víctimas de violencia presentan riesgo de presentar depresión posparto en comparación a las que no fueron maltratadas. Asimismo, se encontró diferencias estadísticas entre depresión posparto y violencia psicológica $(p<0,001)$ y violencia durante la gestación $(p=0,002)$. Existió 5,5 (IC: $<5,5 ; 16,7>$ ) más riesgo de sufrir depresión en aquellas puérperas víctimas de la violencia durante la gestación.

\section{DISCUSIÓN}

Diversos estudios informan que existe una prevalencia de depresión oscilante entre 10 y $20 \%$ de las mujeres en el puerperio tardío; resultados similares encontramos en el presente estudio, con $17 \%$ de casos ${ }^{(2-4)}$.

A diferencia de la literatura mundial que afirma que existen diferencias estadísticamente significativas entre la presentación de depresión posparto y el nivel socioeconómico, el estado marital, la planificación del embarazo y la tristeza materna, en el presente estudio no se demostró dicha relación. Así como Carter y col. ${ }^{(35)}$, quienes no encontraron relación entre el tipo de parto y la presentación de depresión posparto, en el presente trabajo no se demostró diferencias estadísticas entre dichas variables.

Beck y col. ${ }^{(18)}$ determinaron en un metaanálisis los factores predictores de depresión posparto. Nosotros, dentro de dichos factores, encontramos la autoestima baja, presentación de ansiedad durante la gestación, historia de depresión previa al embarazo, satisfacción marital, eventos estresantes durante la gestación y problemas con el cuidado del bebé. De igual manera, un aporte del presente estudio es la determinación del riesgo existente entre depresión posparto y la presentación de ansiedad durante la gestación, historia de depresión previa al embarazo y problemas por los cuidados del bebé.

Con respecto a las cifras de casos de violencia basada en género, se detectó que $51 \%$ de las participantes del estudio fue víctima de diferentes tipos de violencia en algún momento de su vida, cifra que es menor a la detectada por Távara y col. ${ }^{(25)}$, quienes describieron en una población peruana una prevalencia de $69,3 \%$. Los mismos autores, en otro estudio, describieron que del total de las participantes, 31,9\% sufrió de violencia durante la gestación, cifra muy semejante a la que se encontró en la presente investigación ${ }^{(31)}$. Estas cifras difieren considerablemente con las cifras comunicadas en EEUU, donde la violencia durante la gestación oscila entre 3,9 y $8,3 \%{ }^{(32)}$.

Así mismo, se determinó que existe un riesgo alto de sufrir depresión posparto en aquellas mujeres víctimas de la violencia. Estos resultados concuerdan con los resultados descritos por Leung y col. ${ }^{(30)}$, quienes realizaron un estudio de violencia doméstica y depresión posparto en una comunidad de China, donde detectaron una tasa alta de violencia basada en género, fundamentalmente psicológica, y diferencias estadísticamente significativas con los casos de depresión posparto.

A modo de conclusión, en el presente estudio podemos afirmar que existe un mayor riesgo de padecer depresión posparto en aquellas mujeres que fueron víctimas de los diferentes tipos de violencia, que hay tasas altas de violencia en la mujer y que existen factores de la madre que se relacionan con la presentación de depresión posparto.

Al confrontar las cifras publicadas en algunos estudios de la literatura mundial, se puede concluir que el tema de la pre- 
sente investigación es un serio problema de salud pública, que afecta de diferente manera a todas las sociedades, existiendo un marcado interés por los problemas que genera en la madre, como la disminución de la calidad de vida. De igual manera, el niño no tiene estímulos adecuados que le permitan tener un correcto desarrollo físico y neuropsicológico y, adicionalmente, los problemas maritales pueden llevar al padre a padecer una patología poco descrita, denominada depresión posnatal paterna, que se incrementa de $5 \%$ a 40 o $50 \%$ cuando la madre sufre depresión posparto ${ }^{(4)}$.

\section{REFERENCIAS BIBLIOGRÁFICAS}

1. Vicente B, Rioseco P, Saldivia S, Kohn R, Torres $S$. Prevalencia de trastornos psiquiátricos en Latinoamérica: revisión crítica. Rev Colombiana Psiq. 2005;34(4):506-14.

2. Cassano P, Fava M. Depression and public health: an overview. J Psychosom Res. 2002;53:84957.

3. Kessler R. Epidemiology of women and depression. J Affect Disord. 2003;74 5-13.

4. Lee D, Chung T. Postnatal depression: an update. Best Pract Res Clin Obstet Gynaecol. 2006;20(20):1-9.

5. Noble R. Depression in women. Metabolism. 2005:54(Suppl 1):49-52.

6. Alici-Evcimen Y, Sudak D. Postpartum depression. Prim Care Update Obstet Gynecol. 2003:10(5):2106.

7. Birnbaum H, Leong S, Greenberg P. The economics of women and depression: an employer's perspective. J Affect Disord. 2003;74:15-22.

8. Ryan D, Milis L, Misri N. Depression during pregnancy. Can Fam Physician. 2005;51:108793.

9. Brown E, Varghese F, McEwen B. Association of depression with medical illness: does cortisol play a role? Biol Psychiatry. 2004;55:1-9.

10. Wisner K, Parry B, Piontek C. Postpartum depression. N Engl J Med. 2002;347(3):194-9.
11. Campagne D. The obstetrician and depression during pregnancy. Eur J Obstet Gynecol Reprod Biol. 2004;116:125-30.

12. Pearlstein T. Hormones and depression: What are the facts about premenstrual syndrome, menopause, and hormone replacement therapy? Am J Obstet Gynecol. 1995;173(2): 646-53.

13. Beck C. Postpartum depression. AJN. 2006;106(5):40-50.

14. Shors T, Leuner B. Estrogen-mediated effects on depression and memory formation in females. J Affect Disord. 2003;74:85-96.

15. Lawrie T, Hofmeyr G, De Jager M, Berk M, Paiker J, Viljoen E. The risk of post-natal depression was increased in women receiving a long-acting progestogen contraceptive. Evid Based Obstet Gynecol. 1999;1:85.

16. Abou-Saleh M, Ghubash R, Karim L, Krymski M, Bhai I. Hormonal aspects of postpartum depression. Psychoneuroendocrinol. 1998;23(5):465-75.

17. Stowe Z, Nemeroff C. Women at risk for postpartumonset major depression. Am J Obstet Gynecol. 1995;173(2):639-45.

18. Beck C. Predictors of postpartum depression. An update. Nurs Res. 2001;50(5):275-85.

19. Robertson E, Grace S, Wallington T. Depression and anxiety during pregnancy are strong indicators of postpartum depression. Evid Based Ment Health. 2005;8:21

20. Robertson E, Grace S, Tamara, Wallington T. Stewart D. Antenatal risk factors for postpartum depression: a synthesis of recent literature. Gen Hosp Psychiatry. 2004;26:289-95.

21. Da Silva Moraesa I, Tavares Pinheirob R, Azevedo da Silva R, Lessa Hortac B, Rosa Sousa P, Duarte Fariab A. Prevalence of postpartum depression and associated factors. Ver Saúde Pública. 2006;40(1):1-5.

22. Jadresic E, Nguyen N, Halbreich U. What does Chilean research tell us about postpartum depression (PPD)? J Affect Disord. 2006;20:17.

23. Bloch M, Rotenberg T, Koren D, Klein E. Risk factors for early postpartum depressive symptoms. Gen Hosp Psychiatry. 2006;28:3-8.

24. Tatano, $C$. The effects of postpartum depression on child development: a meta-analysis. Arch Psychiatr Nurs. 1998;XII(1):12-20.
25. Távara L, Zegarra T, Zelayal L, Arias M, Estolaza $N$. Detección de violencia basada en género en tres servicios de atención de salud reproductiva. Ginecol Obstet. 2003;49(1):31-8.

26. Mejía, R. Violencia basada en género. Evid Actual Práct Ambul. 2005;8(5):154-6.

27. Heise L, Ellsberg M, Gottmoeller M. A global overview of gender-based violence. Int I Gynaecol Obstet. 2002;78 (Suppl 1):S5-S14.

28. Tavara L. Sexual violence. Best Pract Res Clin Obstet. 2006;20(3):395-408.

29. Schmuel E, Schenker JG. Violence against women: the physician's role. Eur J Obstet Gynecol Reprod Biol. 1998;80:239-45.

30. Leung W, Kung F, Lam J, Leung J, Ho P. Domestic violence and postnatal depression in a Chinese community. Int J Gynaecol Obstet. 2002;79:15966.

31. Távara L, Zegarra T, Huamaní S, Felix F, EspinozaTarazona K, Chumbe-Ruiz 0, y col. Repercusiones maternas y perinatales de la violencia basada en género. Rev Per Ginecol Obstet. 2007;53(1):107.

32. Morris J, Freda M, Bernstein P. Screening for postpartum depression in an inner-city population. Am J Obstet Gynecol. 2003;188(121):7-9.

33. Smallwood S, Jamieson D, Lindsay M. Diagnosing postpartum depression: Can we do better? Am J Obstet Gynecol. 2002;186(5):899-902.

34. Vega J, Mazzotti S, Campos S. Validación de una versión en español de la escala de depresión postnatal de Edimburgo. Actas Esp Psiquiatr. 2002;30(2):106-11.

35. Carter F, Frampton C, Mulder R. Cesarean section and postpartum depression: a review of the evidence examining the link. Psychosom Med. 2006; 68:321-30.

Manuscrito recibido el 25 de mayo de 2009 y aceptado para publicación el 26 de junio de 2009.

Correspondencia:

Dr. Juan Escobar Montalvo

Jr. Huallaga 986.

Lima 1, Perú

Correo-e: juanma_05@hotmail.com 